\title{
Valve sparing root replacement with the reimplantation technique in patients presenting with acute type A aortic dissections
}

\author{
Joshua C. Grimm, John J. Kelly, Wilson Y. Szeto \\ Division of Cardiovascular Surgery, University of Pennsylvania School of Medicine, Penn Presbyterian Medical Center, Heart \& Vascular Pavilion, \\ Philadelphia, PA, USA \\ Contributions: (I) Conception and design: JC Grimm, WY Szeto; (II) Administrative support: WY Szeto; (III) Provision of study materials or patients: \\ WY Szeto; (IV) Collection and assembly of data: JC Grimm, JJ Kelly; (V) Data analysis and interpretation: All authors; (VI) Manuscript writing: All \\ authors; (VII) Final approval of manuscript: All authors. \\ Correspondence to: Wilson Y. Szeto. Division of Cardiovascular Surgery, University of Pennsylvania School of Medicine, Penn Presbyterian Medical Center, \\ Heart and Vascular Pavilion, $2^{\text {nd }}$ Floor, Suite 2A, 51 N. $39^{\text {th }}$ Street, Philadelphia, PA, 19104, USA. Email: Wilson.Szeto@pennmedicine.upenn.edu.
}

\begin{abstract}
Patients presenting with acute type A aortic dissections (ATAAD) comprise an extremely heterogenous population. While a majority are older and suffer from chronic disease states, a noninsignificant minority are relatively healthy without long standing aneurysmal or aortic valve pathology. Selection of an appropriate proximal and distal operation can affect short- and long-term morbidity and mortality as well as the need for valve reintervention. In regard to management of the root, surgeons must be aggressive when indications for replacement—such as a sinus tear, aneurysmal degeneration, or friable tissue-are evident. In experienced centers, it is reasonable to proceed with a valve-sparing procedure in well selected patients. The risks and benefits of this decision must be considered carefully as a root replacement with either a biologic or mechanical prosthesis is preferred over a poorly executed valve sparing procedure. However, if the latter is approached in a standardized fashion with attention to the patient's overall acuity, cardiac function, and aortic valve leaflet mobility, reproducible outcomes can and should be expected. Accordingly, we present our approach to valve sparing root replacement with the reimplantation technique in ATAAD. The following review includes a case-based discussion and video depiction of our technique, including safe and reliable cannulation methods for bypass, cerebral protection strategies, and a structured approach to critically appraise the valve prior to repair.
\end{abstract}

Keywords: Acute type A aortic dissection (ATAAD); valve sparing root replacement; antegrade cerebral protection $(\mathrm{ACP})$

Received: 07 April 2020; Accepted: 03 June 2020; Published: 20 October 2021.

doi: 10.21037/jovs-20-94

View this article at: http://dx.doi.org/10.21037/jovs-20-94

\section{Case scenario}

A 41-year-old female with a medical history of hypertension and active tobacco use presented to an outside facility with acute onset chest and back pain. A CT angiogram demonstrated an acute type A aortic dissection (ATAAD) that extended from the root to the bilateral iliac arteries with a possible tear at the sinotubular junction. She was promptly transferred straight to the operating room at our hospital where a transesophageal echocardiogram (TEE) confirmed the findings of the CT scan. Notably, she had a low-normal ejection fraction, a dissection extending to the right and non-coronary cusps, and involvement of the innominate and right common carotid arteries with preserved flow. The following narrative and associated video (Video 1) will outline our approach to a valve sparing root replacement with the reimplantation technique.

\section{Preoperative assessment}

Prior to sternotomy, the CT scan and TEE are reviewed 


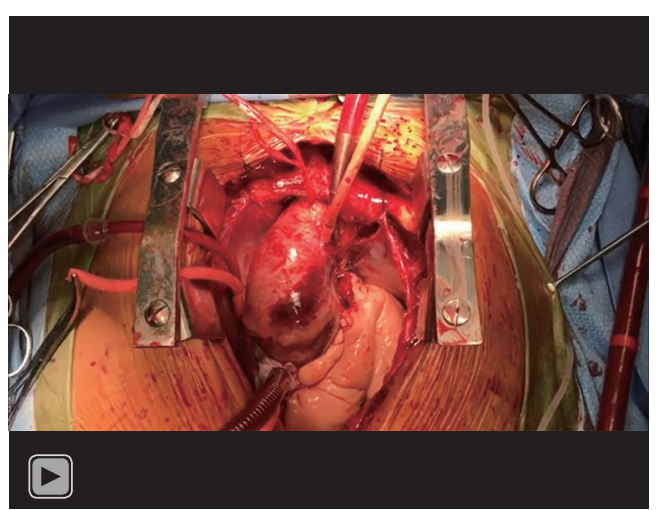

Video 1 The following video illustrates our technique for a valve sparing root procedure (reimplantation) in patients presenting with acute type A aortic dissection.

to characterize the root and arch anatomy in addition to the cardiac function and any valvulopathies present. It is critical to identify abnormalities such as an aberrant right subclavian which could be catastrophic if missed during right axillary cannulation for antegrade cerebral protection (ACP). In regards to aortic valve function, a valve sparing approach is undoubtedly simplified in cases of isolated central regurgitation with near normal leaflet motion (El Khoury type I) as complex cusp repairs might be ill-advised in the context of an ATAAD. An astute anesthesiologist can provide the surgeon with critical information regarding the nature of the dissection and possible involvement of the sinus segment, valve, and coronary arteries. Patients are excluded from a valve-sparing procedure if they are of advanced age, present in extremis with a complex dissection or have poor cardiac function.

\section{Cannulation strategy}

There are a variety of techniques that can be employed in order to safely institute cardiopulmonary bypass in patients presenting with an ATAAD (1). As aforementioned, the CT scan will not only provide relevant information regarding the entry tear and relationship between the true and false lumens but it will also recognize involvement of peripheral vessels which might be utilized for cannulation (i.e., axillary or femoral vessels). Alternatively, the aorta can be cannulated directly over a wire with the assistance of TEE $(1,2)$. Following successful cannulation, the aorta is cross-clamped and the heart arrested with a combination of retrograde and direct ostial cardioplegia. The patient is then cooled to a core temperature of $28^{\circ} \mathrm{C}$.

\section{Evaluation of the root}

If arrest is not obtained with administration of ostial cardioplegia, or if the patient presents with ECG consistent with myocardial ischemia, coronary malperfusion $(\sim 7 \%$ of cases) should be immediately suspected. The most commonly utilized classification system includes: type A lesions in which the dissection creates a false lumen in close proximity to the coronary ostium, type B lesions in which the false lumen extends into the actual body of the coronary artery, and, finally, type C lesions in which there is an evulsion of the coronary artery (3). Repair strategies span from sandwich repair with inside and outside sutures in type A lesions to bypass grafting in type C lesions (3). Regardless of whether the root requires an intervention or not, recognizing a coronary injury is critical to ensure appropriate myocardial protection throughout the operation.

Often times, despite a dissection extending into the sinus of Valsalva, the root can be managed with valve resuspension, felt neo-media reconstruction, and supracoronary tube graft placement (in addition to any required arch interventions) (4). If, however, the patient presents with an aneurysm of, or primary dissection tear in, the sinus segment or a syndrome with a predilection towards root degeneration (i.e., Marfan's), a definitive root procedure should be considered.

In cases in which the valve is not salvageable (i.e., chronic fenestrations or calcifications, commissural disruption, or leaflet pathology), it is best to proceed with a composite valve graft reconstruction. Otherwise, a valve-sparing root replacement can be entertained. The following will outline the reimplantation technique as initially described by Dr. David.

\section{Preparation and dissection of the root}

In order to access the free margin length, cusp mobility, and coaptation, traction sutures are placed above each commissure (will be utilized later to re-suspend the functional aortic annulus inside the Dacron graft). The root is then circumferentially dissected down to the ventriculoaortic junction, which can be challenging in ATAAD as there is typically dense hematoma due to local perforation. Each sinus segment is excised leaving $8-10 \mathrm{~mm}$ of residual aortic tissue attached to the annulus and commissures. 


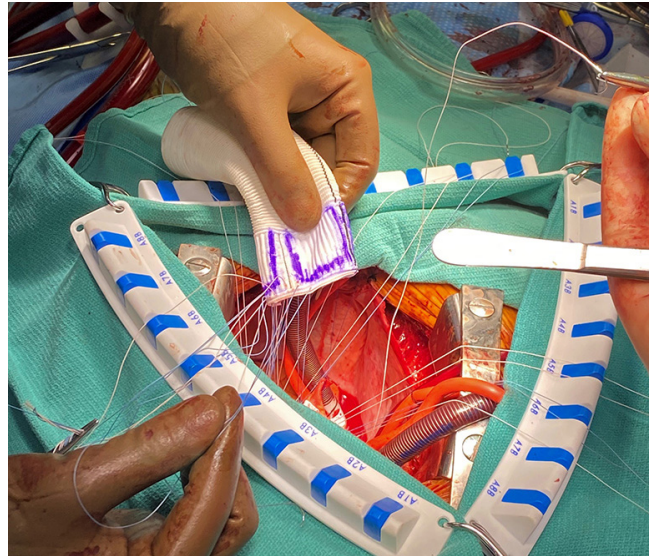

Figure 1 Primary suture line. Four sets of horizontal mattress sutures are placed 4-6 mm below the annulus in each sinus segment. Care is taken to avoid spanning the nadir or the commissure with a stitch. Sutures are then passed through the skirt of the Dacron graft at a distance that parallels their placement in the root.

Coronary buttons are fashioned with adequate aortic tissue remaining around the ostia. The leaflet geometric height (from insertion in the annulus at its nadir to the tip of the leaflet edge) is then assessed and divided by 0.7 . This value should roughly correlate to the annular dimension obtained with a Freestyle sizer (Medtronic, Minneapolis, MN) and approximate the annular diameter needed for appropriate leaflet coaptation. If there is a major discrepancy between the two, additional leaflet work, such as plication, might be necessary. To select the appropriate sized Valsalva graft (Terumo, Sunrise, FL), a value of five is added to the annular diameter as measured by the sizer. In cases of baseline aortic valve insufficiency, it is recommended to downsize rather than upsize the graft.

\section{Primary suture line}

Once the appropriate graft has been selected, the primary suture line is constructed with a braided suture, approximately $4-6 \mathrm{~mm}$ below the annulus, roughly at the level of the ventriculo-aortic junction. The stitches are placed as a horizontal mattress from inside of the aorta out. In trileaflet valves, 4 stitches are placed equidistantly in each sinus segment being mindful to never span the nadir or a commissure within a stitch. The skirt of the graft is then trimmed, leaving 1 ring below the 'Valsalva' portion of the Dacron. The graft is then notched at the left-right commissural junction to accommodate the right ventricular

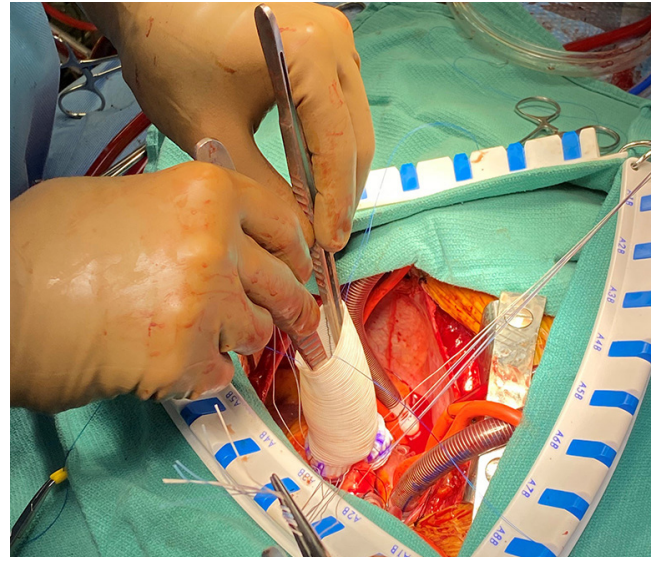

Figure 2 Dacron graft secured to the root. Radial tension is placed on the primary sutures and the Dacron graft is lowered towards the root. The commissural stitches are pulled through the middle of the graft and the primary sutures are sequentially tied.

outflow tract and the sutures are placed inside out at the most proximal aspect of the graft (Figure 1). The graft is then lowered towards the ventriculo-aortic junction by applying equal tension on all of the sutures. The stitches are then sequentially tied, securing the graft below the annulus, with care not to tear the muscle (Figure 2).

\section{Special considerations in bicuspid valves}

For the most part, the major principles of a valve sparing root procedure in a trileaflet valve also translate to the unique situation of a bicuspid aortic valve (BAV). Typically, the annulus is slightly larger in BAV cases, but the Dacron graft is sized similarly to as described above. The BAV classification has been described extensively, but two important considerations are worth noting-Sievers type 0 and type 1 valves (5). In the former, 4 horizontal mattress sutures are placed below each sinus segment, at the level of the ventriculo-aortic, to comprise the primary suture line. In the latter, 4 horizontal mattress sutures are placed below the reference leaflet and 4 below the fused leaflet with 2 being placed on each side of the raphe. It is important to note that with Sievers type 0 bicuspid valves, the leaflets can 'billow' despite a coaptation plan above the annulus. It is unclear what, if any, impact this will have on long-term valve durability. The secondary suture line is completed in an identical fashion as outlined above.

If there is lack of coaptation following valve-resuspension, a variety of repair strategies can be entertained. If there 

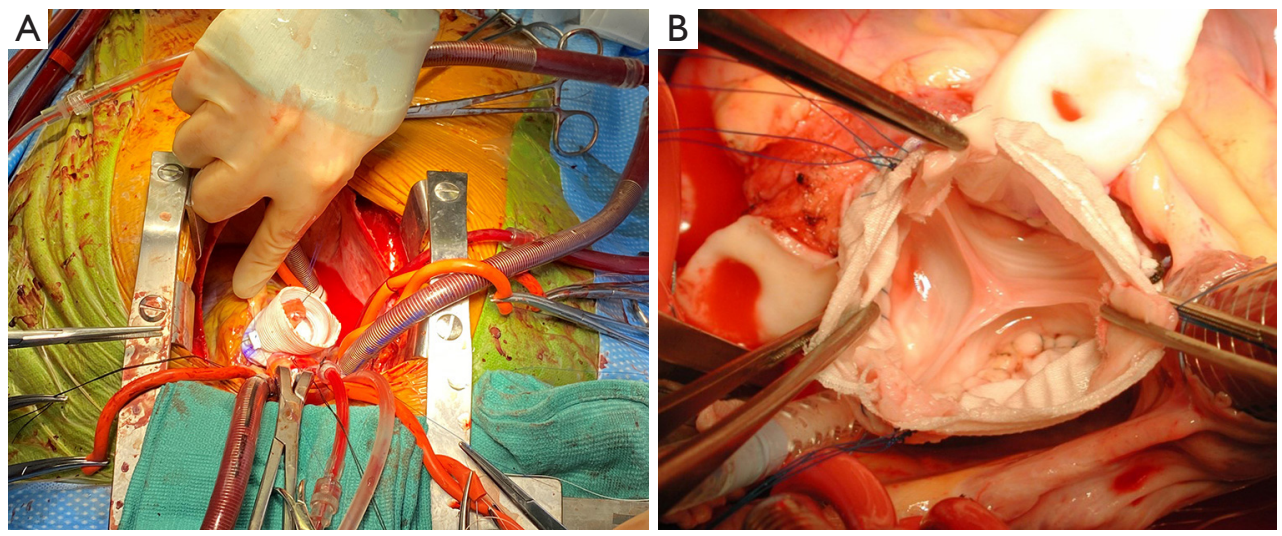

Figure 3 Secondary suture line. (A) Three sets of double-armed prolene sutures are placed through the graft and residual aortic tissue starting at the nadir of each cusp. A rough schematic of the secondary suture line is constructed on the graft material to use as a guide when placing each stitch. (B) Each suture is continued towards its corresponding commissure where it is secured, effectively creating six independent suture lines.

is excess free margin (prolapse) and a coaptation plane below the reference point, a central plication or triangular resection can be entertained. In cases of a restricted leaflet with a fibrotic raphe, the dense tissue can be shaved or resected and closed to augment mobility. Some centers have described bovine pericardial patch repair of valves with large pseudocommissures but these techniques should only be employed by experienced surgeons, especially in the context of an ATAAD (6).

\section{Valve resuspension and secondary suture line}

Tension is applied to the previously placed commissural sutures to determine the appropriate resuspension height required to ensure valve competence and they are tied in place (typically correlates to the sinotubular portion of the Valsalva graft). The secondary suture line is initiated at each nadir with a 4-0 prolene suture and carried towards the corresponding commissure ( 3 sets of doublearmed sutures, Figure 3). The leaflet tissue is carefully manipulated to ensure an adequate zone of coaptation. If redundant tissue is appreciated, the cusps can be plicated and the valve re-examined. It is prudent to test valve competence with saline.

\section{Coronary artery reimplantation}

A burner is employed to create a generous defect in the Valsalva graft, and the left coronary button is secured with a 5-0 prolene suture. Volume is left in the heart in order to locate the appropriate spot on the graft for creation of the right coronary anastomosis.

\section{Arch intervention}

Once the core temperature reaches $28^{\circ} \mathrm{C}$, circulatory arrest is initiated and the patient is exsanguinated. Of note, EEG will be symmetrical but not silenced. The distal ascending aorta and arch are inspected carefully in order to identify any tear sites. If the innominate is not dissected, a $9 \mathrm{Fr}$ cardioplegia cannula is placed directly into the artery. If the tear extends up the cervical vessels, antegrade cerebral perfusion is administered with a balloon tipped catheter. Depending upon the extent of arch involvement, the appropriate distal procedures is performed with felt neo-media reconstruction of the dissected aorta. The graft and cerebral circulation are de-aired, cardiopulmonary bypass is re-instituted, and a cross-clamp is applied (7). A graft-to-graft anastomosis is then performed to create an ascending aortic length of 5-6 cm without distortion of the root which might adversely affect valve competence or coronary perfusion (Figure 4).

\section{Comments}

ATAAD confers considerable morbidity and mortality as well as a unique set of complex surgical challenges $(8,9)$. While both the proximal and distal aorta require a strategic approach, the former is germane to this discussion. As aforementioned, there are several options to manage the proximal aorta in order to mitigate short- and long- 


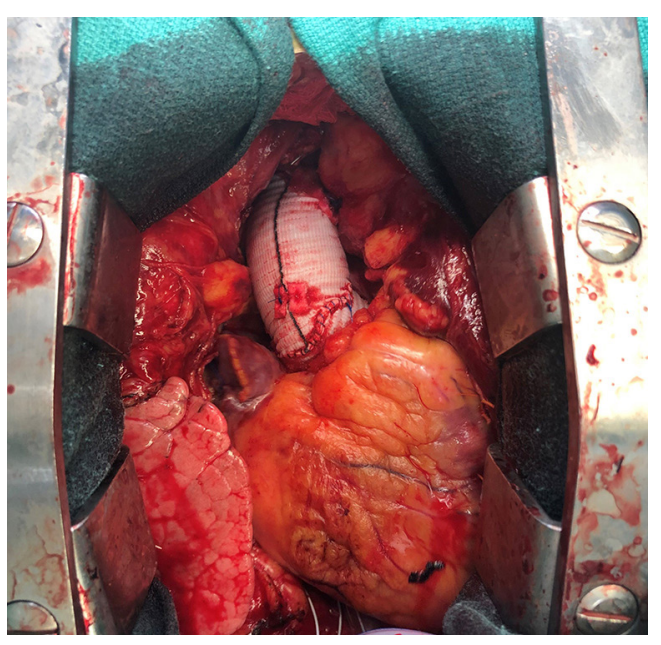

Figure 4 Valve sparing root reconstruction and hemiarch replacement. Following circulatory arrest and the necessary arch replacement, the distal and proximal Dacron segments are anastomosed.

term complications, such as rupture and re-operation. The appropriate strategy depends on the extent of sinus/ valve involvement, baseline characteristics of the root, and surgeon familiarity with advanced repair strategies. Multiple prior studies have demonstrated that the need for a root replacement does not alter mortality in this cohort of patients $(10,11)$. The valve-sparing approach offers a unique platform for a durable repair without the inherent limitations of a composite valve graft and can be reliably performed with excellent outcomes in high volume centers (12-15).

In a recent report, Tanaka and colleagues present their findings of 328 consecutive patients undergoing repair of an ATAAD. Approximately 9\% (30 patients) required a root replacement and, in a majority, the valve was successfully spared (24 patients). The authors had an aggressive approach to pursuing a valve-sparing procedure and were not deterred by fenestrations or commissural disruption. Freedom from $\geq$ moderate aortic insufficiency and valve reintervention were both approximately $82 \% \pm 10 \%$ at 5 years. Nearly half of the patients presented with detached commissures, which were repaired with buttressed sutures and, in most, either GRF glue or BioGlue. Interestingly, 3/7 patients treated with GRF glue re-presented for commissural detachment and required reoperation (12). Similar results were evident in a study of ATAAD patients by Leshnower et al. (13), who demonstrated a slightly higher incidence of root interventions (40\%) overall. In the cohort of patients undergoing a valve-sparing procedure, $90 \%$ and
$94 \%$ had $\leq 1+$ aortic insufficiency upon chest closure and on follow-up (mean 40 months), respectively. Moreover, no patients required subsequent valve replacement. Subramanian compared results amongst ATAAD patients managed with composite valve grafts and one of two valve sparing techniques (reimplantation or remodeling). There was no statistical difference in perioperative or 5-year mortalities and the need for valve reintervention was only $7.5 \%$ in the valve sparing cohorts at a mean follow-up of 44 months (14).

In order to ensure excellent results in this challenging population, an algorithmic approach must be exacted. First, the proximal aorta must be managed appropriately with either a repair (valve resuspension and felt neomedia) or a replacement (composite valve graft or valve sparing procedure). The decision to proceed with a reimplantation must be weighed against the probability of a successful operation given the presence of fenestrations, cusp retractions/calcifications, commissural pathology or eccentric jets.

\section{Acknowledgments}

Funding: None.

\section{Footnote}

Provenance and Peer Review: This article was commissioned by the Guest Editors (Ibrahim Sultan and George Arnaoutakis) for the series "Advancement in the Surgical Treatment of Aortic Dissection" published in Fournal of Visualized Surgery. The article has undergone external peer review.

Conflicts of Interest: All authors have completed the ICMJE uniform disclosure form (available at https://jovs. amegroups.com/article/view/10.21037/jovs-20-94/coif). The series "Advancement in the Surgical Treatment of Aortic Dissection" was commissioned by the editorial office without any funding or sponsorship. WYS reports nonfinancial support from Edwards Lifesciences, Medtronic, and Terumo Aortic, outside the submitted work. The authors have no other conflicts of interest to declare.

Ethical Statement: The authors are accountable for all aspects of the work in ensuring that questions related to the accuracy or integrity of any part of the work are appropriately investigated and resolved. Written informed 
consent was obtained from the patient for publication of the case portion of this review article and any accompanying images.

Open Access Statement: This is an Open Access article distributed in accordance with the Creative Commons Attribution-NonCommercial-NoDerivs 4.0 International License (CC BY-NC-ND 4.0), which permits the noncommercial replication and distribution of the article with the strict proviso that no changes or edits are made and the original work is properly cited (including links to both the formal publication through the relevant DOI and the license). See: https://creativecommons.org/licenses/by-nc-nd/4.0/.

\section{References}

1. Kreibich M, Chen Z, Rylski B, et al. Outcome after aortic, axillary, or femoral cannulation for acute type A aortic dissection. J Thorac Cardiovasc Surg 2019;158:27-34.e9.

2. Frederick JR, Yang E, Trubelja A, et al. Ascending aortic cannulation in acute type a dissection repair. Ann Thorac Surg 2013;95:1808-11.

3. Kreibich M, Bavaria JE, Branchetti E, et al. Management of Patients With Coronary Artery Malperfusion Secondary to Type A Aortic Dissection. Ann Thorac Surg 2019;107:1174-80.

4. Bavaria JE, Brinster DR, Gorman RC, et al. Advances in the treatment of acute type A dissection: an integrated approach. Ann Thorac Surg 2002;74:S1848-52; discussion S1857-63.

5. Sievers HH, Schmidtke C. A classification system for the bicuspid aortic valve from 304 surgical specimens. J Thorac Cardiovasc Surg 2007;133:1226-33.

6. Arnaoutakis GJ, Sultan I, Siki M, et al. Bicuspid aortic valve repair: systematic review on long-term outcomes.

doi: 10.21037 /jovs-20-94

Cite this article as: Grimm JC, Kelly JJ, Szeto WY. Valve sparing root replacement with the reimplantation technique in patients presenting with acute type A aortic dissections. J Vis Surg 2021;7:42.
Ann Cardiothorac Surg 2019;8:302-12.

7. Sultan I, McGarvey J, Vallabhajosyula P, et al. Routine use of hemiarch during acute type A aortic dissection repair. Ann Cardiothorac Surg 2016;5:245-7.

8. Berretta P, Patel HJ, Gleason TG, et al. IRAD experience on surgical type A acute dissection patients: results and predictors of mortality. Ann Cardiothorac Surg 2016;5:346-51.

9. Dib B, Seppelt PC, Arif R, et al. Extensive aortic surgery in acute aortic dissection type A on outcome - insights from 25 years single center experience. J Cardiothorac Surg 2019;14:187.

10. Yang B, Norton EL, Hobbs R, et al. Short- and longterm outcomes of aortic root repair and replacement in patients undergoing acute type A aortic dissection repair: Twenty-year experience. J Thorac Cardiovasc Surg. 2019;157:2125-36.

11. Chiu P, Trojan J, Tsou S, et al. Limited root repair in acute type A aortic dissection is safe but results in increased risk of reoperation. J Thorac Cardiovasc Surg 2018;155:1-7.e1.

12. Tanaka H, Ikeno Y, Abe N, et al. Outcomes of valvesparing root replacement in acute Type A aortic dissection. Eur J Cardiothorac Surg 2018;53:1021-6.

13. Leshnower BG, Myung RJ, McPherson L, et al. Midterm results of David $V$ valve-sparing aortic root replacement in acute type A aortic dissection. Ann Thorac Surg 2015;99:795-800; discussion 800-1.

14. Subramanian S, Leontyev S, Borger MA, et al. Valvesparing root reconstruction does not compromise survival in acute type A aortic dissection. Ann Thorac Surg 2012;94:1230-4.

15. Sultan I, Komlo CM, Bavaria JE. How I Teach a Valve-Sparing Root Replacement. Ann Thorac Surg 2016;101:422-5. 